ARTICLE

https://doi.org/10.1038/s41467-019-12877-0

\title{
Modification of boron nitride nanocages by titanium doping results unexpectedly in exohedral complexes
}

\author{
Ruyi $\mathrm{Li}^{1} \&$ Yang Wang (i) ${ }^{1 \star}$
}

Despite their early experimental production and observation, the unambiguous molecular structures of metal-containing boron nitride (BN) nanocages still remain mysterious. It has been commonly assumed that this family of compounds has the metal atom confined inside the cage, just like their isoelectronic cousins, carbon metallofullerenes do. Here, we demonstrate that $\mathrm{Ti}(\mathrm{BN})_{n}(n=12-24)$ complexes have, unexpectedly, an exohedral structure instead of an endohedral one, which could be verified by collision-induced dissociation experiments. The predicted global minimum structures exhibit some common bonding features accounting for their high stability, and could be readily synthesized under typical conditions for generating BN nanoclusters. The Ti doping dramatically changes not only the cage topology, but the arrangement of $\mathrm{B}$ and $\mathrm{N}$ atoms, endowing the resultant compounds with potential for $\mathrm{CO}_{2}$ capture and nitrogen fixation. These findings may expand or alter the understanding of BN nanostructures functionalized with other transition metals. 
$s$ the closest isoelectronic analog of carbon, boron nitride (BN) can likewise be shaped into a great diversity of nanoforms in various dimensions, such as nanocages, nanotubes, nanosheets, and nanoporous frameworks ${ }^{1-4}$. These thermally and chemically highly stable compounds have a wide range of potential applications in material ${ }^{3-5}$ and biomedical ${ }^{6,7}$ sciences. BN fullerenes, in particular, have been synthesized by electron irradiation ${ }^{8,9}$ or arc-melting ${ }^{10,11}$ methods, with their chemical compositions and cage-like structures identified by time-of-flight mass spectrometry and transmission electron microscopy. They are envisioned as promising materials for hydrogen storage ${ }^{12,13}$, metal-free catalysis ${ }^{14}$, molecular sensors ${ }^{15}$, and natural gas separation ${ }^{16}$.

In a stable $\mathrm{BN}$ cage, $\mathrm{B}$ and $\mathrm{N}$ atoms are alternately connected so that only $\mathrm{N}-\mathrm{B}$ bonds are present and relatively much weaker $\mathrm{N}-\mathrm{N}$ or $\mathrm{B}-\mathrm{B}$ bonds $^{17}$ are avoided. The consequent equal stoichiometric numbers for $\mathrm{B}$ and $\mathrm{N}$ were confirmed by electron energy loss spectroscopy ${ }^{9}$. For the same reason, unlike carbon fullerenes whose cage framework is usually built of pentagons and hexagons, a $\mathrm{BN}$ fullerene consists of squares, hexagons ${ }^{18}$, and sometimes octagons for larger cages ${ }^{19}$. The presence of any oddmembered rings, like pentagons or heptagons, would inevitably introduce $\mathrm{N}-\mathrm{N}$ and $\mathrm{B}-\mathrm{B}$ bonds. Among a large number of possible isomers, the energetically most favorable $\mathrm{BN}$ cages tend to have all squares staying away from each other so as to minimize the strain energy induced by contact of these small rings. This leads to the so-called isolated square rule ${ }^{18}$, an analog of the wellknown isolated pentagon rule for carbon fullerenes ${ }^{20}$. Recently, much attention has been paid to the intriguing fact that this latter rule is often violated when a carbon cage encapsulates a metal atom or a metal-containing cluster, due to strong metal-cage interactions ${ }^{21-23}$. More generally, the observed cage topology (viz., the connectivity between atoms) of many endohedral carbon metallofullerenes differs dramatically from that of the corresponding neutral empty fullerenes ${ }^{21-23}$. A natural question arises: whether the same behavior occurs when a $\mathrm{BN}$ fullerene combines with a metal atom.

Experimentally, BN cages containing a single metal atom have been produced by arc-melting synthesis, where the formation of $\mathrm{Fe}(\mathrm{BN})_{36}{ }^{24}, \mathrm{La}(\mathrm{BN})_{36}{ }^{25}$ and $\mathrm{Y}(\mathrm{BN})_{n}(n=36,37,48)^{11}$ species was confirmed by mass spectrometry and high-resolution electron microscopy. However, due to lack of crystal structure determination, the unambiguous, atomically resolved structures of metal-doped BN fullerenes still remain unclear. The same problem was encountered for relatively small carbon metallofullerenes $\mathrm{MC}_{2 n}(\mathrm{M}=\mathrm{Ti}, \mathrm{Zr}, \mathrm{U} ; n=14-25)$, which were detected in gas-phase mass spectra ${ }^{26}$. Nevertheless, with the aid of quantum chemistry calculations, the molecular structures of $\mathrm{Ti} @ \mathrm{C}_{2 n}$ have been convincingly identified 27 . Among them, the abovementioned modification of cage form upon combining with the metal was also manifested ${ }^{27}$. One important but perhaps overlooked fact is that all Ti-doped carbon fullerenes are endohedral complexes with the metal atom confined inside the cage. This conclusion has been extended, by implication, to BN metallofullerenes, and the observed molecules in experiments were interpreted as endohedral species ${ }^{11,24,25}$. Although unproven, it has even become a commonly accepted premise upon which many studies on metal-doped BN cages are based ${ }^{28-31}$. As a matter of fact, the electron microscopy images for metalcontaining $\mathrm{BN}$ cages ${ }^{11,24,25}$ were two dimensional projections of nanostructures ${ }^{30}$, and therefore could not really tell whether the metal stayed inside or outside of the cage.

In this work, we show that BN fullerenes doped with a single $\mathrm{Ti}$ atom in general have an exohedral structure, at least for cage sizes comparable to their experimentally observed carbon counterparts, Ti@ ${ }_{2 n}{ }^{26}$. DFT calculations clearly demonstrate that the externally bound complexes of $\mathrm{Ti}(\mathrm{BN})_{n}$ are strikingly more stable than the endohedral ones, by an energy of the order of $100 \mathrm{kcal} \mathrm{mol}^{-1}$. As strongly contrasted with carbon fullerenes, it is highly exergonic for a $\mathrm{BN}$ cage to attach a $\mathrm{Ti}$ atom from outside, whereas encaging it is totally hindered from a thermodynamic point of view. This suggests that exohedral $\operatorname{Ti}(\mathrm{BN})_{n}$ complexes can most likely be produced by high-temperature synthesis ${ }^{32,33}$ like arcmelting, where global minimum structures on the free energy surface are usually formed. Furthermore, changes in cage structure take place upon doping with a single $\mathrm{Ti}$ atom, even more drastic than the case of carbon fullerenes. The doped cage contains a few pentagons and even heptagons, the unwelcome rings that are always absent in a pristine BN fullerene. As we will see, such a variation in cage framework, along with notable rearrangement of $\mathrm{B}$ and $\mathrm{N}$ atoms, allows for a maximum metal-cage interaction, featured by the formation of four strong $\mathrm{Ti}-\mathrm{N}$ bonds. a

$(\mathrm{BN})_{19}$

$2 \mathrm{~B}-\mathrm{B}$ and $2 \mathrm{~N}-\mathrm{N}$ bonds

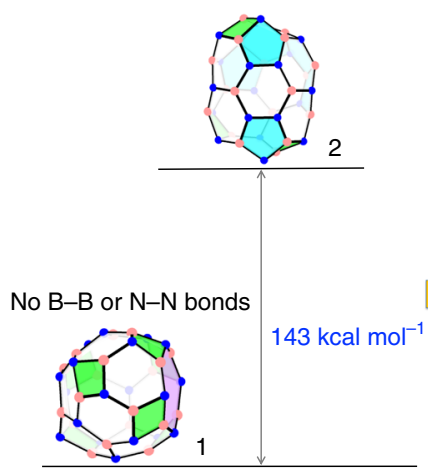

b

$\mathrm{Ti}(\mathrm{BN})_{19}$
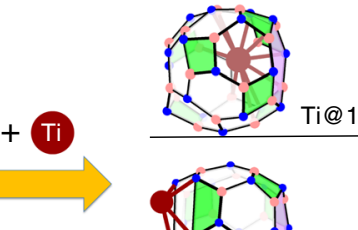

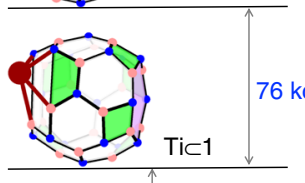

$28 \mathrm{kcal} \mathrm{mol}^{-1}$

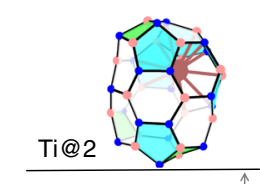

$203 \mathrm{kcal} \mathrm{mol}^{-1}$

Fig. 1 Relative energies of two isomers of pristine cages $(B N)_{19}$ and of their Ti-doped complexes. a Isomer $\mathbf{1}$ is the lowest energy form of (BN) ${ }_{19}$, consisting of 7 squares, 13 hexagons, and 1 octagon, with no B-B or N-N bond present. Isomer 2 contains 3 squares, 6 pentagons, and 12 hexagons. The presence of two B-B and two N-N bonds makes the latter isomer lie significantly higher in energy than the former. $\mathbf{b}$ Conversely, when doped with a single Ti atom, cage $\mathbf{2}$ becomes much more stable than cage $\mathbf{1}$, and gives the global minimum structure of $\mathrm{Ti}(\mathrm{BN})_{19}$. For both cages, the exohedral complex, $\mathrm{Ti} \subset(\mathrm{BN})_{19}$, has substantially lower energy than the corresponding endohedral one, Ti@(BN) 19 . All relative energies (including zero-point energy correction) have been obtained from DFT calculations. Boron, nitrogen, and titanium atoms are indicated by pink, blue, and dark red circles, respectively. Squares, pentagons, hexagons, and octagons are shown in green, cyan, white, and magenta, respectively 
Based on the understanding of the extraordinary stability of these uncovered complexes, some simple topological rules are established, enabling us to find, among a vast number of possibilities, the most stable structures of Ti-doped BN fullerenes that are likely to be observed in future experiments.

\section{Results}

Global minimum structures of $\mathbf{T i}(\mathbf{B N})_{19}$. We have considered the possibility of doping $(\mathrm{BN})_{n}$ cages with a Ti atom, given the fact that so far only a single $\mathrm{Ti}, \mathrm{Zr}$, or $\mathrm{U}$ atom has been experimentally inserted into small carbon fullerenes ${ }^{26}$. The choice of $\mathrm{Ti}$ would be of particular interest in practice, since its nontoxic nature permits safe experiments for macroscopic synthesis $^{26}$. To start off, we selected a medium-sized $\mathrm{Ti}(\mathrm{BN})_{19}$, considering the size range of experimentally observed Ti@ $\mathrm{C}_{2 n}$ $(n=14-25)$, for a thorough investigation. According to the present and previous ${ }^{19}$ DFT calculations (see Methods), the lowest energy isomeric form of prinstine $(\mathrm{BN})_{19}$ is cage $\mathbf{1}$ (see Fig. 1a), which consists of 7 squares, 13 hexagons, and 1 octagon, with alternately linked $\mathrm{B}$ and $\mathrm{N}$ atoms. In comparison, cage isomer 2 shown in Fig. 1a contains 3 squares, 6 pentagons, and 12 hexagons and has thus, inevitably, two $\mathrm{B}-\mathrm{B}$ and two N-N bonds. As expected, the latter cage is significantly less stable (by $143 \mathrm{kcal} \mathrm{mol}^{-1}$ ) than the former. However, when they are doped with a Ti atom the situation is reversed: the lowest energy isomer of $\mathrm{Ti}(\mathrm{BN})_{19}$ is the one with the $\mathrm{Ti}$ atom bound outside cage 2 (hereafter symbolized by $\mathrm{Ti} \subset 2$ ), while isomer $\mathrm{Ti} \subset \mathbf{1}$ lies 28 $\mathrm{kcal} \mathrm{mol}^{-1}$ higher than Ti $\subset \mathbf{2}$, as shown in Fig. 1b. Yet, a more unexpected observation is that for both cages, binding a Ti atom outside the cage is substantially more favorable in energy than encapsulating the metal inside; as shown in Fig. 1b, the relative energy of the endohedral Ti@ $(\mathrm{BN})_{19}$ with respect to the corresponding exohedral $\mathrm{Ti} \subset(\mathrm{BN})_{19}$ is as high as 76 and $203 \mathrm{kcal}$ $\mathrm{mol}^{-1}$, respectively, for cage $\mathbf{1}$ and $\mathbf{2}$. This is a remarkably surprising result since it is well-known that carbon metallofullerenes are generally endohedral complexes with enclosed metal atoms or clusters (at least for cage sizes as small as $\mathrm{C}_{28}$ ), ${ }^{21,26}$ and for this reason it has been commonly assumed that their $\mathrm{BN}$ analogs have likewise an endohedral structure $11,24,25,28-31$.

By a systematic and exhaustive search of low-energy isomers of $\mathrm{Ti}(\mathrm{BN})_{19}$ (see Methods and Supplementary Methods 1-3), we have found, aside from the aforementioned $\mathrm{Ti} \subset \mathbf{2}$, other two global minimum structures, $\mathrm{Ti} \subset \mathbf{3}$ and $\mathrm{Ti} \subset \mathbf{4}$ (see Fig. 2a). The energy difference between the three is less than $0.7 \mathrm{kcal} \mathrm{mol}^{-1}$. Although differing in cage topology (cage 4 even has a heptagon, see the Schlegel diagrams in Fig. 2a), the three isomers share some common bonding features responsible for their high stability. As shown in Fig. 2a, the Ti atom is exohedrally bonded to the four N atoms located on a hexagonal ring, which, before combined with $\mathrm{Ti}$, has two N-N bonds on the parallel sides and four B-N bonds on the other (for simplicity, hereafter we refer to such a hexagon as the key hexagon). Upon doping, the two parallel $\mathrm{N}-\mathrm{N}$ bonds break up so as to form four tetragonal pyramid-shaped Ti-N bonds (with $\mathrm{N}-\mathrm{Ti}-\mathrm{N}$ angles of about $75^{\circ}$ and $90^{\circ}$ ). Note that the cleavage of $\mathrm{N}-\mathrm{N}$ bonds enables the $\mathrm{N}$ atoms to stably coordinate to the Ti while maintaining the delocalized $\pi$ bonding between $\mathrm{N}$ and $\mathrm{B}$ atoms. Because of the two N-N linkages on the key hexagon, the global minimum structures have inevitably two B-B bonds in the rest part of the cage (see the bonds in red color in Fig. 2a), whose strength is much weaker and therefore destabilizes the system to some extent. Nonetheless, this destabilization is surpassed by the formation of four strong Ti-N bonds. The Ti-N bond lengths in $\mathrm{Ti} \subset 2$ are 1.95-1.98 Å, even slightly shorted than those in the $\mathrm{TiN}_{n}^{+}$clusters recently produced by laser ablation and observed by mass spectrometry. ${ }^{34}$ Besides, additional stability is

\begin{tabular}{|c|c|c|c|c|c|c|c|}
\hline Isomer $^{a}$ & $\mathbf{R E}^{\mathbf{b}}$ & $\mathbf{R}_{\mathbf{1}}{ }^{\mathrm{c}}$ & $\mathbf{R}_{\mathbf{2}}{ }^{\mathbf{c}}$ & $\mathbf{R}_{\mathbf{3}}{ }^{\mathbf{c}}$ & $\mathbf{R}_{\mathbf{4}}{ }^{\mathbf{c}}$ & $\mathbf{R}_{5}{ }^{\mathbf{c}}$ & $\mathbf{R}_{6}{ }^{\mathbf{c}}$ \\
\hline $\mathrm{Ti} \subset \mathbf{2}$ & 0.0 & 5 & 5 & 6 & 6 & 6 & 6 \\
\hline $\mathrm{Ti} \subset \mathbf{3}$ & 0.3 & 4 & 5 & 6 & 6 & 6 & 6 \\
\hline $\mathrm{Ti} \subset \mathbf{4}$ & 1.1 & 5 & 5 & 6 & 6 & 5 & 7 \\
\hline $\mathrm{Ti} \subset \mathbf{5}$ & 3.4 & 5 & 5 & 6 & 6 & 6 & 6 \\
\hline $\mathrm{Ti} \subset \mathbf{6}$ & 5.9 & 4 & 4 & 6 & 6 & 6 & 6 \\
\hline $\mathrm{Ti} \subset \mathbf{7}$ & 6.1 & 5 & 5 & 6 & 5 & 6 & 6 \\
\hline $\mathrm{Ti} \subset \boldsymbol{8}$ & 6.7 & 5 & 5 & 6 & 6 & 6 & 6 \\
\hline $\mathrm{Ti} \subset \mathbf{9}$ & 8.0 & 5 & 5 & 6 & 5 & 6 & 6 \\
\hline Ti $\subset \mathbf{1 0}$ & 8.8 & 5 & 5 & 6 & 6 & 6 & 5 \\
\hline Ti $\subset \mathbf{1 1}$ & 9.1 & 5 & 5 & 6 & 6 & 6 & 6 \\
\hline Ti $\subset \mathbf{1 2}$ & 9.3 & 5 & 5 & 6 & 6 & 6 & 6 \\
\hline $\mathrm{Ti} \subset \mathbf{1 3}$ & 9.6 & 4 & 5 & 6 & 5 & 6 & 6 \\
\hline $\mathrm{Ti} \subset \mathbf{1 4}$ & 9.7 & 5 & 5 & 6 & 6 & 6 & 6 \\
\hline $\mathrm{Ti} \subset \mathbf{1 5}$ & 9.8 & 5 & 5 & 6 & 6 & 7 & 5 \\
\hline
\end{tabular}

gained in the complex owing to the reduced strain energy, as the modified cage by Ti doping (form 2) contains more pentagons and less squares than the pristine one (form 1, see Fig. 1).

Structural and bonding features of stable $\mathrm{Ti}(\mathrm{BN})_{19}$ isomers. A closer inspection of the neighboring rings surrounding the key hexagon reveals more rules for determining low energy $\mathrm{Ti}(\mathrm{BN})_{n}$ isomers. By examining nearly 2500 possible candidate structures of $\mathrm{Ti} \subset(\mathrm{BN})_{19}$ containing a key hexagon, we have found that all low-lying isomers comply with the following rules. First, the neighboring rings that share an $\mathrm{N}-\mathrm{N}$ side with the key hexagon (i.e., the top and bottom rings shown in Fig. $2 b, R_{1}$ and $R_{2}$ ) are either a square or a pentagon. The strain induced by these smaller rings can facilitate the opening of the $\mathrm{N}-\mathrm{N}$ bonds and hence the formation of $\mathrm{Ti}-\mathrm{N}$ bonds. Moreover, the other four neighboring rings of the key hexagon (i.e., the lateral rings shown in Fig. 2b, $R_{3}-R_{6}$ ) prefer to be a pentagon, or a hexagon, or a heptagon. The larger, less strained lateral rings seem to help sustain the N-B bonds in the key hexagon upon hosting a $\mathrm{Ti}$ atom. As shown in Table 1, these structural characteristics are clearly evidenced in the low-lying isomers of $\mathrm{Ti} \subset(\mathrm{BN})_{19}$ (within $10 \mathrm{kcal} \mathrm{mol}^{-1}$ with respect to the global minimum). Many more examples are provided in Supplementary Note 3 and Supplementary Table 6.

To gain further understanding of why the key hexagon is essential for the stability of $\mathrm{Ti}(\mathrm{BN})_{n}$ complexes, we have analyzed orbital interactions between the metal and the cage, based on the maximum bonding fragment orbital approach ${ }^{35,36}$. Figure 3 presents the orbital correlation diagram for the lowest energy complex, $\mathrm{Ti} \subset 2$, revealing four principal metal-cage bonding interactions (each with a Wiberg bond order $^{37}$ greater than 0.5 ). We can see that the three leading interactions $\left(\sigma_{1}, \pi_{1}\right.$, and $\left.\pi_{2}\right)$ are between Ti's $d$ orbitals $\left(d_{x y}, d_{y z}\right.$, and $d_{x z}$, respectively) and the cage's symmetry-adapted group orbitals, the latter coming mostly from the four $\mathrm{N}$ atoms of the key hexagon. The last major bonding, $\sigma_{2}$, is resulted from the $s d^{3}$ hybridized orbital of Ti interacting with the group orbital from the $\mathrm{N}$ atoms. The significant values of bond orders (about 0.6-0.8) for these interactions indicate four typical polarized covalent bonds ${ }^{35}$ formed between the metal and the cage, reflecting the maximum bonding ability acquired by the tetravalent $\mathrm{Ti}$, which would account for the high stability of the complex Ti $\subset \mathbf{2}$. Further, in order to achieve a maximum overlap between the fragment orbitals, especially as required by the symmetry of the strongest 
a

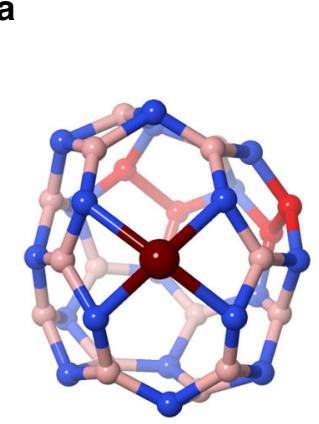

$\mathrm{Ti} \subset 2$

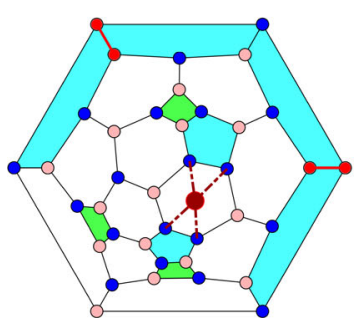

$\mathrm{Ti}(\mathrm{BN})_{19}$

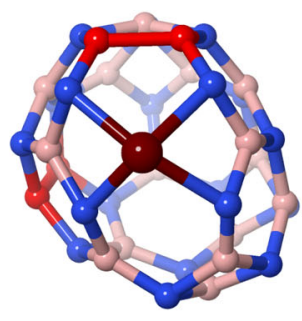

$\mathrm{Ti} \subset 3$
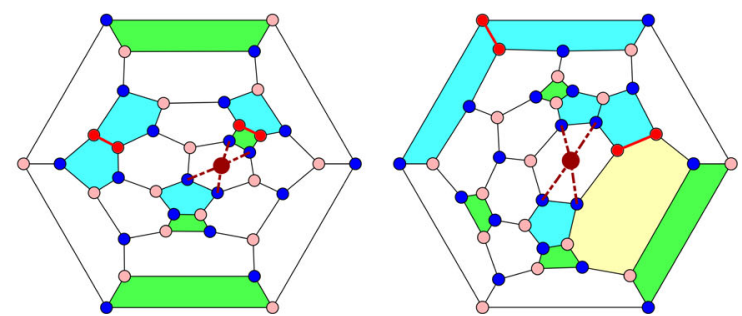

b

Key structural pattern for stable $\mathrm{Ti}(\mathrm{BN})_{n}$

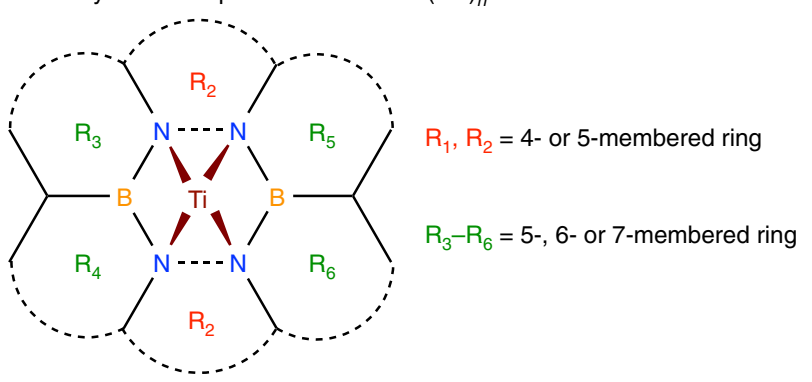

Fig. 2 Global minimum energy structures of Ti(BN) ${ }_{19}$, revealing common bonding features. a Molecular structures (upper panel) and Schlegel diagrams (lower panel) of the three global minimum isomers (almost degenerate within an energy difference of $0.7 \mathrm{kcal} \mathrm{mol}^{-1}$ ). Boron, nitrogen, and titanium atoms are depicted in pink, blue, and dark red, respectively. The two B-B bonds in each molecule are highlighted in red. In Schlegel diagrams, Ti-N bonds are indicated by dashed lines, and squares, pentagons, hexagons, and heptagon are shown in green, cyan, white, and yellow, respectively. $\mathbf{b}$ The key structural pattern present in all stable Ti-doped $(B N)_{n}$ cages. The Ti atom is located above a hexagonal ring, where four $\mathrm{N}$ atoms sit on two parallel sides and two $\mathrm{B}$ atoms at opposite corners. To achieve higher stability, the top and bottom rings $\left(R_{1}\right.$ and $\left.R_{2}\right)$ adjacent to the doped hexagon are preferably squares or pentagons, while the four lateral neighboring rings $\left(\mathrm{R}_{3}-\mathrm{R}_{6}\right)$ opt for pentagons, hexagons, or heptagons

bonding orbital, $\sigma_{1}$, the four Ti-N bonds adopt preferably a tetragonal pyramidal conformation, as mentioned above and illustrated in Fig. 2. It is worth noting that, in accord with the aufbau principle ${ }^{35}$, the effective oxidation number of $\mathrm{Ti}$ in this compound is +4 , as expected for this group 4 transition metal.

Isomers with less than four $\mathrm{Ti}-\mathrm{N}$ bonds are much higher in energy, such as Ti $\subset \mathbf{1}$ shown in Fig. 1 b (see Supplementary Note 4 and Supplementary Fig. 5 for more examples), since a Ti-N bond is almost twice as strong as a Ti-B bond (their bond energy being 114 and $65 \mathrm{kcal} \mathrm{mol}^{-1}$ (ref. ${ }^{17}$ ), respectively). Furthermore, binding the $\mathrm{Ti}$ atom with four $\mathrm{N}$ atoms from a square or a pentagon will introduce more energetically unfavorable $\mathrm{N}-\mathrm{N}$ and B-B bonds (see Fig. 4a, b, where the number of these bonds, $\lambda$, is indicated), which would significantly destabilize the system. Likewise, we can rule out the possibility of adding the $\mathrm{Ti}$ to a hexagon site where the four $\mathrm{N}$ atoms are consecutively placed, as indicated in Fig. 4c. As for additions to a heptagonal or octagonal ring, although it is possible to host a tetracoordinated $\mathrm{Ti}$ while maintaining only two N-N/B-B connections (see Fig. 4e, f), calculations show that $\mathrm{Ti} \subset(\mathrm{BN})_{19}$ with such a heptagon (octagon) ligand lie at least $22.5(85.1) \mathrm{kcal} \mathrm{mol}^{-1}$ in energy above the global minimum (see Supplementary Figs. 6 and 7 for more examples). The main reason is that a larger ring like heptagon or octagon is more flexible and thus more easily subject to deformation induced by the strain in the cage. Consequently, the four Ti-N bonds deviate noticeably from the ideal tetragonal pyramidal configuration, with distorted bond angles and the four $\mathrm{N}$ atoms out of coplanarity (see Supplementary Note 5). Based on the above discussion, it is reasonable to speculate that the key hexagon provides a chemically favorable site to accommodate a $\mathrm{Ti}$ atom, constituting the essential feature of stable Ti-doped BN cages.

At this point, we can propose simple structural rules for stable Ti-doped BN cages, as follows: (1) the cage contains one key hexagon with appropriate neighboring rings to host the $\mathrm{Ti}$ atom, as described in Fig. 2b; (2) it has only two B-B bonds; and (3) it obeys the isolated square rule ${ }^{18}$. Violations to the latter two rules lead to higher energy isomers, as exemplified in Supplementary Tables 7 and 8 . By applying these rules to all possible cage isomers of $(\mathrm{BN})_{19}$ (including regioisomers with different arrangement of B and $\mathrm{N}$ atoms), we ended up with 1065 candidates. Subsequent prescreening and refinement DFT calculations allowed us to predict the lowest energy isomers of $\operatorname{Ti}(\mathrm{BN})_{19}$ that could be produced in experiments. For 
thermodynamically controlled gas-phase generation such as under arc-melting conditions $s^{11,24,25}$, we have predicted the temperature-dependent mole fractions of major products of

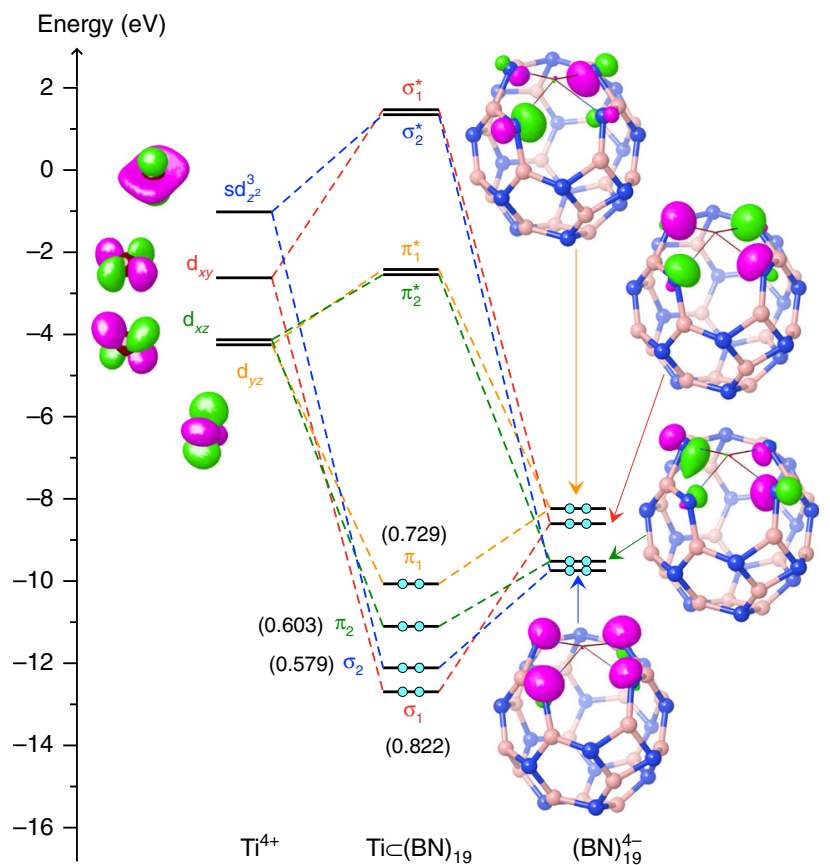

Fig. 3 Orbital correlation diagram unraveling the nature of the metal-cage bonding in $\mathrm{Ti} \subset \mathbf{2}$. The analysis is based on the maximum bonding fragment orbital approach ${ }^{35,36}$. Each of the Ti's atomic orbitals (left panel) is paired up with one of the cage's fragment orbitals (right panel), leading to a pair of bonding and anti-bonding orbitals of the complex (middle panel). The energy levels of the associated fragment orbitals and complex orbitals are connected by dashed lines in the same color. Electrons are shown by cyan filled circles. Orbital surfaces are depicted using an isovalue of 0.10 . The Wiberg bond orders ${ }^{37}$ in parentheses indicate that all these four bonding interactions are essentially covalent. According to the aufbau principle 35 , this orbital diagram suggests an oxidation state of +4 for $\mathrm{Ti}$
$\operatorname{Ti}(\mathrm{BN})_{19}$ on the basis of Maxwell-Boltzmann distribution ${ }^{38}$ (see Supplementary Note 8). For clarity, Fig. 5a only shows the relative concentrations of six major products (from $\mathrm{Ti} \subset \mathbf{2}$ to $\mathrm{Ti} \subset \mathbf{7}$, labeled in ascending order of energy), along with that of isomer $\mathrm{Ti}$ $\subset \mathbf{1}$ for comparison. Their relative energies are presented in Table 2 and molecular structures are shown in Supplementary Fig. 12. At typical temperature range $(1500-2500 \mathrm{~K})$ for producing $\mathrm{BN}$ nanocages ${ }^{32,33}$, isomer $\mathrm{Ti} \subset \mathbf{4}$ with a heptagonal ring is the most abundant product, followed by $\mathrm{Ti} \subset 3$. Despite being the lowest energy isomer at $0 \mathrm{~K}, \mathrm{Ti} \subset \mathbf{2}$ is only the third major product at synthesis temperatures up to $\sim 2400 \mathrm{~K}$. Beyond that temperature, other relatively higher energy isomers (especially $\mathrm{Ti} \subset 7$ ) become more competitive. In comparison, the product formed with the lowest energy pristine cage, $\mathrm{Ti} \subset \mathbf{1}$ (indicated by the dashed line in Fig. 5a), would be obtained in a very low yield during the whole temperature range of interest.

Synthetic viability and possible experimental verification. Guided by the stability rules as an efficient prescreening tool, we have discovered the lowest energy structures of $\mathrm{Ti}(\mathrm{BN})_{n}$ of other cage sizes (see Methods and Supplementary Method 3 for detailed procedures). These structures are summarized in Supplementary Note 6 and Supplementary Figs. 8-13. The temperaturedependent mole fractions for their major products are presented in Supplementary Figs. 15-19. Similar to the case of Ti $(\mathrm{BN})_{19}$, all exohedral complexes are considerably more stable than the endohedral ones, even for cages as large as $(\mathrm{BN})_{24}$, as can be seen in Table 2. To explore the viability of their synthesis, we have computed, at arc-melting temperatures and a pressure of 1 atm, the Gibbs free energies of complexation, $\Delta G_{c}$, defined as

$$
\Delta G_{\mathrm{c}}=G\left[\mathrm{Ti}(\mathrm{BN})_{n}\right]-G[\mathrm{Ti}]-G\left[(\mathrm{BN})_{n}\right],
$$

where $G\left[\mathrm{Ti}(\mathrm{BN})_{n}\right], G[\mathrm{Ti}]$, and $G\left[(\mathrm{BN})_{n}\right]$ are the Gibbs free energy of the complex $\operatorname{Ti}(\mathrm{BN})_{n}$, of a single $\mathrm{Ti}$ atom and of the pristine cage $(\mathrm{BN})_{n}$, respectively. Note that we have taken, depending on the given temperature, the lowest free energy isomer for $(\mathrm{BN})_{n}$ and for $\operatorname{Ti}(\mathrm{BN})_{n}$, which may differ from the lowest energy form at $0 \mathrm{~K}$. The solid circles in Fig. $5 \mathrm{~b}$ present the complexation free energies of $\operatorname{Ti}(\mathrm{BN})_{n}$ for $n=12,13,14,16,19$, and 24 at a

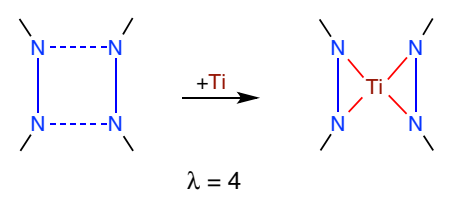<smiles>CB1N(C)N2N(C)B(C[18F])N(C)[In]2N1C</smiles>

$\lambda=3$

e<smiles>CB1B(C)N(C)N(C)B(C)N(C)N1C</smiles>

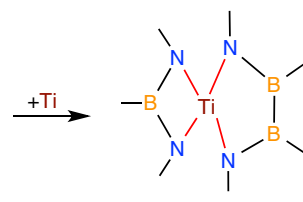

$\lambda=2$

b

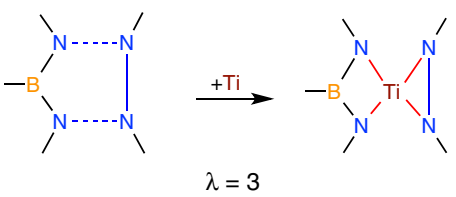<smiles>CB1N(C)N(C)B(C)N(Cl)N1C</smiles>

Key hexagon

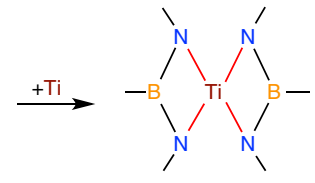

$\lambda=2$<smiles>[3H]N1B(C)B(C)N(C)N(C)B(C)B(C)N(C)B2N(C)B(C)N(C)B(N2C)N1C</smiles>

$\lambda=2$

Fig. $4 \mathrm{~N}-\mathrm{N}$ bond cleavage and Ti-N bond formation schemes upon Ti doping of a BN cage. The additions of a Ti atom to the following faces of the BN cage are considered: a A square with $\lambda=4$, where $\lambda$ denotes the number of N-N/B-B bonds. $\mathbf{b} A$ pentagon with $\lambda=3$. $\mathbf{c} A$ hexagon with four $N$ atoms in tandem $(\lambda=3)$. $\mathbf{d}$ A key hexagon with two parallel $N-N$ bonds $(\lambda=2)$. e A heptagon with two separated $N-N$ bonds $(\lambda=2)$. $\mathbf{f}$ An octagon with two parallel $N-N$ bonds $(\lambda=2)$. The $\mathrm{N}-\mathrm{N}$ bonds to be broken are indicated by blue dashed lines, while the subsequently formed Ti-N bonds are shown by red solid lines 

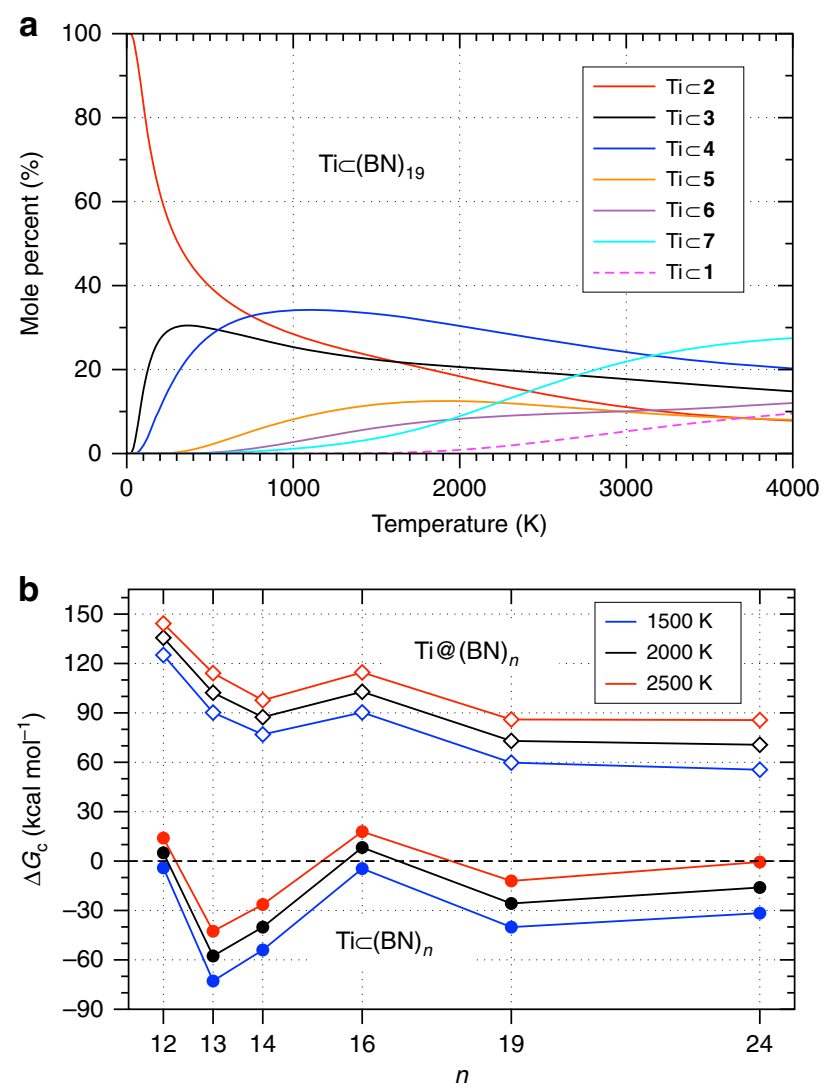

Fig. 5 Relative concentrations and complexation free energies of $T i(B N)_{n}$ at various temperatures. a DFT predicted mole fractions of the major products by a thermodynamically controlled synthesis of $\mathrm{Ti}(\mathrm{BN})_{19}$, as a function of temperature up to $4000 \mathrm{~K}$. The solid lines correspond to the six lowest energy isomers and the dashed line denotes the isomer with cage form $\mathbf{1}$. b Gibbs free energies of complexation of $\operatorname{Ti}(B N)_{n}(n=12,13,14,16,19,24)$, $\Delta G_{c^{\prime}}$ defined by Eq. (1) in the text, at 1500,2000 , and $2500 \mathrm{~K}$ (indicated in blue, black, and red, respectively), the typical temperatures for producing BN nanocages 32,33 . Solid circles and empty diamonds represent, respectively, the exohedral and endohedral complexes. Note that the exohedral products presented here are the lowest Gibbs free energy isomer at a given temperature, while the endohedral products have the cage form that corresponds to the lowest energy isomer of pristine cages (see Supplementary Note 7, Supplementary Table 9 and Supplementary Fig. 14 for specific structures). All results in $\mathbf{a}$ and $\mathbf{b}$ are obtained at a pressure of $1 \mathrm{~atm}$ temperatures 1500,2000 , and $2500 \mathrm{~K}$. As we can see, all $\mathrm{Ti} \subset$ $(\mathrm{BN})_{n}$ complexes can be spontaneously formed at all these temperatures except for $n=12$ and 16, whose synthesis may not be ruled out at lower temperatures like $1500 \mathrm{~K}$, or at a higher pressure $^{33,39}$. Notably, the production of $\mathrm{Ti} \subset(\mathrm{BN})_{13}$ and $\mathrm{Ti} \subset$ $(\mathrm{BN})_{14}$ are highly exergonic $\left(\Delta G_{\mathrm{c}}=-57.6\right.$ and $-40.1 \mathrm{kcal} \mathrm{mol}^{-1}$ at $2000 \mathrm{~K}$, respectively). The reason is that their pristine cage reactants are exceptionally unstable, for it is mathematically impossible to have a cage form satisfying the isolated square rule for $(\mathrm{BN})_{13}$ and $(\mathrm{BN})_{14}$ (ref. $\left.{ }^{18}\right)$. This implies that these otherwise elusive $\mathrm{BN}$ clusters can be readily stabilized by doping with a single $\mathrm{Ti}$ atom.

In direct contrast, Fig. 5b also depicts the complexation free energies of endohedral products made of the lowest energy pristine cage, which are apparently all strongly endergonic at arcmelting temperatures. By comparison, their carbon counterparts exhibit the opposite behavior: for instance, at $2000 \mathrm{~K}$ and $1 \mathrm{~atm}$, the DFT calculated $\Delta G_{c}=-3.8$ and $-37.7 \mathrm{kcal} \mathrm{mol}^{-1}$ for Ti $\subset$ $\mathrm{C}_{38}$ and $\mathrm{Ti} @ \mathrm{C}_{38}$ (ref. ${ }^{27}$ ), respectively. This supports the fact that endohedral metallofullerenes are generally observed in arcdischarge production ${ }^{21,26}$, whereas the formation of exohedral species is usually precluded for carbon fullerenes unless by direct attachment of the metal to a preformed cage at relatively low temperatures ${ }^{40}$. The striking contrast between carbon and $\mathrm{BN}$ fullerenes is probably due to the following facts. In a nonpolar, homonuclear carbon fullerene, the frontier $\pi$ electrons are delocalized over the whole cage, preferring to form an also delocalized, multicenter bond with the Ti atom. And by binding the metal inside the comparatively isotropic cage, a maximum coordination number is achieved and so is maximum stability. On the other hand, a BN fullerene consists of polar bonds with much more localized valence electrons. The Ti atom seems to go for the more electronegative, $\mathrm{N}$ atoms other than the $\mathrm{B}$ atoms, leading to four Ti-N bonds, as allowed by this tetravalent metal. Since the metal-cage bonding is essentially localized, an exohedral configuration is favored, probably in order to minimize strain energy and/or to maximize orbital overlaps.

To confirm the exohedral structure of $\mathrm{Ti}(\mathrm{BN})_{n}$ complexes, collision-induced dissociation experiments could be carried out. Early such experiments ${ }^{40}$ unequivocally confirmed that the single metal atom in $\mathrm{MC}_{60}^{+}(\mathrm{M}=\mathrm{Fe}, \mathrm{Co}, \mathrm{Ni}, \mathrm{Cu}, \mathrm{Rh}, \mathrm{La})$ was externally bound to the carbon cage, as a result of direct detachment of the metal at relatively low excitation energies. In the present case of exohedral $\operatorname{Ti}(\mathrm{BN})_{n}$, collisional excitation or photoexcitation would give rise to $\mathrm{Ti}^{+}$ions, leaving the $\mathrm{BN}$ cage neutral and intact, since the ionization potential of the latter (e.g., $8.2 \mathrm{eV}$

\begin{tabular}{|c|c|c|c|c|c|c|}
\hline Isomer & $\mathrm{Ti}(\mathrm{BN})_{12}$ & $\operatorname{Ti}(\mathrm{BN})_{13}$ & $\mathrm{Ti}(\mathrm{BN})_{14}$ & $\mathrm{Ti}(\mathrm{BN})_{16}$ & $\mathrm{Ti}(\mathrm{BN})_{19}$ & $\mathrm{Ti}(\mathrm{BN})_{24}$ \\
\hline Ti@1 & 123.6 & 169.9 & 138.7 & 92.3 & 103.4 & 88.2 \\
\hline $\mathrm{Ti} \subset \mathbf{2}$ & 0.0 & 0.0 & 0.0 & 0.0 & 0.0 & 0.0 \\
\hline Ti@2 & 187.7 & 297.1 & 267.1 & 212.1 & 202.7 & 182.6 \\
\hline $\mathrm{Ti} \subset \mathbf{5}$ & 5.9 & 17.6 & 12.9 & 10.4 & 3.2 & 2.0 \\
\hline $\mathrm{Ti} \subset \mathbf{6}$ & 7.3 & 18.8 & 13.3 & 10.5 & 5.7 & 2.7 \\
\hline $\mathrm{Ti} \subset \mathbf{7}$ & 8.0 & 19.1 & 14.1 & 11.0 & 5.9 & 4.6 \\
\hline $\mathrm{Ti} \subset \boldsymbol{8}$ & 8.2 & 19.7 & 15.2 & 11.6 & 6.2 & 5.5 \\
\hline$T i \subset \mathbf{9}$ & 8.8 & 20.0 & 15.9 & 12.1 & 7.9 & 5.5 \\
\hline $\mathrm{Ti} \subset \mathbf{1 0}$ & 9.1 & 20.3 & 17.1 & 12.2 & 8.3 & 6.0 \\
\hline
\end{tabular}


for $(\mathrm{BN})_{13}$ by DFT) is much higher than that of $\mathrm{Ti}(6.6 \mathrm{eV})$. Accordingly, one should detect only $\mathrm{Ti}^{+}$ions in mass spectra, by using an excitation energy of the order of $10 \mathrm{eV}$ (e.g., the computed dissociation energy of $\left[\mathrm{Ti} \subset(\mathrm{BN})_{13}\right]^{+}$being $10.7 \mathrm{eV})$. Otherwise, if the metal atom is enclosed in the cage, much higher excitation energy would be required to take out the metal, and the fragmented pieces of parent cage might be observed as well.

In conclusion, we have found that doping $\mathrm{BN}$ fullerenes with a single Ti atom generally results in exohedral complexes instead of endohedral ones, which is unexpectedly contrary to the case of most carbon metallofullerenes. Inserting a metal atom into a fullerene molecule needs to break first the chemical bonds of the cage, requiring a sufficiently hot environment so that the metal is being enclosed during the formation of its host cage. However, DFT calculations show that at such high temperatures encapsulation of a $\mathrm{Ti}$ atom into a $\mathrm{BN}$ nanocage is thermodynamically inhibited, although it might be accomplished by resort to molecular surgery 41,42 . Conversely, exohedrally doped BN fullerenes exhibit high thermodynamic stability that makes their synthesis viable at high temperatures typical for yielding BN nanocages. We suggest that the exohedral nature of $\operatorname{Ti}(\mathrm{BN})_{n}$ clusters could be proved experimentally by studying the collisioninduced dissociation ${ }^{40}$ or dissociation following photoexcitation of the complexes.

The doping with a single $\mathrm{Ti}$ atom can change profoundly not only the cage topology, but also the arrangement of $\mathrm{B}$ and $\mathrm{N}$ atoms. As opposed to pristine $\mathrm{BN}$ cages consisting of evenmembered rings to avoid any $\mathrm{B}-\mathrm{B}$ or $\mathrm{N}-\mathrm{N}$ contacts, Ti-doped cages incorporate also pentagons and contain two B-B bonds, in order to acquire maximum bonding interaction with $\mathrm{Ti}$. The energy cost paid by introducing two relatively weak B-B bonds is compensated and surpassed by the formation of four strong Ti-N bonds, as well as by the alleviation of strain in the doped cage compared to the pristine one. Interestingly, these $\mathrm{B}-\mathrm{B}$ antisites have been shown to have potential for $\mathrm{CO}_{2}$ capture $^{43}$ and nitrogen fixation ${ }^{44}$ at ambient conditions.

In view of the present results for Ti-doped BN nanocages, it is conceivable to expect similar or different implications for other BN nanostructures functionalized with other transition metals. For instance, preliminary calculations suggest that different transition metals may behave differently in modifying the structures of $\mathrm{BN}$ nanocages, which opens an interesting topic for future research. We hope our findings will stimulate further experimental investigations to generate, identify, and characterize this particular family of compounds.

\section{Methods}

DFT calculations. All DFT calculations were performed using the Gaussian 16 suite of programs ${ }^{45}$ at the B3LYP/6-31+G(d) level, including Grimme's D3 dispersion correction with Becke-Johnson damping ${ }^{46}$. The accuracy of basis set was verified by additional calculations using the Def2-TZVP and Def2-TZVPP basis sets (see Supplementary Note 1 and Supplementary Tables 2 and 3). We also carried out some single-point calculations with the double-hybrid B2PLYP-D3/ Def2-TZVP method and obtained similar relative energies and complexation energies (see Supplementary Note 2 and Supplementary Tables 4 and 5). All geometries were optimized without any constraint and confirmed to be minima on the potential energy surface via vibrational frequency analysis. For low-lying energy isomers, both singlet and triplet electronic states were taken into account, and the stability of wavefunction was verified. Relative energies were computed including zero-point energy correction.

Procedures of search for global minimum structures. Topologies of all BN cages were constructed by the plantri program ${ }^{47}$, and then converted to atomic Cartesian coordinates by the embed program in the $\mathrm{CaGe}$ package ${ }^{48}$. Cage isomers with distinct atomic connectivity and regioisomers with distinct $\mathrm{B}$ and $\mathrm{N}$ distribution are uniquely identified based on the canonical labeling of atoms ${ }^{49}$ following the breadth-first-search numbering algorithm ${ }^{50}$. We systematically determined the lowest energy isomers of pristine cages, which all have alternately linked $\mathrm{B}$ and $\mathrm{N}$ atoms. To enumerate possible candidates for cages containing a key hexagon, we optimized the arrangement of $\mathrm{B}$ and $\mathrm{N}$ atoms and attained all possible isomers having the minimum number (namely two) of B-B bonds (see Supplementary Method 2 and Supplementary Figs. 1 and 2 for algorithm). The subsequent Ti $\subset$ $(\mathrm{BN})_{n}$ complexes were calculated using the 6-31G(d) (or 3-21G for $n=48$ ) basis set (see Supplementary Figs. 3 and 4 for justification of using the 6-31G(d) basis set), allowing us to rule out a great number of high energy isomers. For endohedral complexes Ti@(BN $)_{n}$, as well as Ti $\subset(\mathrm{BN})_{n}$ made of the lowest energy pristine cage, we explored all possible initial structures by placing the $\mathrm{Ti}$ atom at different faces of the cage and determined the corresponding lowest energy isomer. Preliminary tests showed that the $\mathrm{Ti}$ atom bound at vertex or bond center sites are energetically less favorable than at face centers. The detailed prescreening procedure is described in Supplementary Method 3, with the total number of considered structures summarized in Supplementary Table 1.

\section{Data availability}

The authors declare that the data supporting the findings of this study are available within the paper and its supplementary information files.

Received: 30 July 2019; Accepted: 27 September 2019; Published online: 28 October 2019

\section{References}

1. Golberg, D. et al. Boron nitride nanotubes and nanosheets. ACS Nano 4, 2979-2993 (2010)

2. Arenal, R. \& Lopez-Bezanilla, A. Boron nitride materials: an overview from 0d to 3d (nano)structures. Wiley Interdiscip. Rev. Comput. Mol. Sci. 5, 299-309 (2015).

3. Pakdel, A., Bando, Y. \& Golberg, D. Nano boron nitride flatland. Chem. Soc. Rev. 43, 934-959 (2014)

4. Weng, Q., Wang, X., Wang, X., Bando, Y. \& Golberg, D. Functionalized hexagonal boron nitride nanomaterials: emerging properties and applications. Chem. Soc. Rev. 45, 3989-4012 (2016).

5. Shtansky, D. V., Firestein, K. L. \& Golberg, D. V. Fabrication and application of bn nanoparticles, nanosheets and their nanohybrids. Nanoscale 10, 17477-17493 (2018)

6. Merlo, A., Mokkapati, V. R. S. S., Pandit, S. \& Mijakovic, I. Boron nitride nanomaterials: biocompatibility and bio-applications. Biomater. Sci. 6, 2298-2311 (2018).

7. Ribeiro, H., Luciano, M. A., C, P. V. R., Vilela, D. N. \& Andrade, L. M. Functionalized boron nitride applications in biotechnology [online first]. In Recent Advances in Boron-Containing Materials (ed. Aydin, M.) (IntechOpen, 2019).

8. Stéphan, O. et al. Formation of small single-layer and nested BN cages under electron irradiation of nanotubes and bulk material. Appl. Phys. A 67, 107-111 (1998).

9. Golberg, D., Bando, Y., Stéphan, O. \& Kurashima, K. Octahedral boron nitride fullerenes formed by electron beam irradiation. Appl. Phys. Lett. 73, 2441-2443 (1998).

10. Oku, T. et al. Synthesis, atomic structures and properties of carbon and boron nitride fullerene materials. Mater. Sci. Eng. B 74, 206-217 (2000).

11. Oku, T., Nishiwaki, A. \& Narita, I. Formation and atomic structures of bnnn ( $\mathrm{n}=24-60)$ clusters studied by mass spectrometry, high-resolution electron microscopy and molecular orbital calculations. Physica B 351, 184-190 (2004).

12. Lian, G. et al. Controlled fabrication of ultrathin-shell BN hollow spheres with excellent performance in hydrogen storage and wastewater treatment. Energy Environ. Sci. 5, 7072-7080 (2012).

13. Ganguly, G., Malakar, T. \& Paul, A. In pursuit of sustainable hydrogen storage with boron-nitride fullerene as the storage medium. ChemSusChem 9, 1386-1391 (2016).

14. Esrafili, M. D., Nematollahi, P. \& Nurazar, R. A comparative study of the co oxidation reaction over pristine and c-doped boron nitride fullerene. RSC Adv. 6, 17172-17178 (2016).

15. Chigo-Anota, E., Escobedo-Morales, A. \& Hernández-Cocoletzi, H. Nitric oxide adsorption on non-stoichiometric boron nitride fullerene: structural stability, physicochemistry and drug delivery perspectives. Physica E 74, 538-543 (2015).

16. Sun, Q., Sun, C., Du, A. \& Li, Z. Charged-controlled separation of nitrogen from natural gas using boron nitride fullerene. J. Phys. Chem. C 118, 30006-30012 (2014)

17. Luo, Y. R. Bond dissociation energies. In CRC Handbook of Chemistry and Physics (CRC Press/Taylor and Francis, Boca Raton, 2009). 
18. Fowler, P. W., Heine, T., Mitchell, D., Schmidt, R. \& Seifert, G. Boron-nitrogen analogues of the fullerenes: the isolated-square rule. J. Chem. Soc. Faraday Trans. 92, 2197-2201 (1996).

19. Gan, L.-H., Li, R. \& An, J. The structures and stability of BNNN clusters with octagon(s). RSC Adv. 2, 12466-12473 (2012).

20. Kroto, H. W. The stability of the fullerenes $c_{n}$, with ${ }_{n}=24,28,32,36,50,60$ and 70. Nature 329, 529-531 (1987).

21. Popov, A. A., Yang, S. \& Dunsch, L. Endohedral fullerenes. Chem. Rev. 113, 5989-6113 (2013).

22. Rodríguez-Fortea, A., Alegret, N., Balch, A. L. \& Poblet, J. M. The maximum pentagon separation rule provides a guideline for the structures of endohedral metallofullerenes. Nat. Chem. 2, 955-961 (2010).

23. Wang, Y., Díaz-Tendero, S., Alcamí, M. \& Martín, F. Cage connectivity and frontier $\pi$ orbitals govern isomer relative stability of fullerene anions and cations. Nat. Chem. 7, 927-934 (2015).

24. Oku, T. \& Suganuma, K. High-resolution electron microscopy and structural optimization of c36, b36n36 and fe@b36n36 clusters. Diam. Relat. Mater. 10, 1205-1209 (2001).

25. Oku, T., Kuno, M. \& Narita, I. High-resolution electron microscopy and electronic structures of endohedral la@b36n36 clusters. Diam. Relat. Mater. 11, 940-944 (2002).

26. Dunk, P. W. et al. The smallest stable fullerene, $\mathrm{m} @ \mathrm{c}_{28}(\mathrm{~m}=\mathrm{ti}, \mathrm{zr}, \mathrm{u})$ : stabilization and growth from carbon vapor. J. Am. Chem. Soc. 134, 9380-9389 (2012).

27. Mulet-Gas, M. et al. Small endohedral metallofullerenes: exploration of the structure and growth mechanism in the ti@c $2 n(2 n=26-50)$ family. Chem. Sci. 6, 675-686 (2015).

28. Wang, J., Ma, L., Zhao, J., Wang, B. \& Wang, G. Stability and magnetic properties of transition metal atoms endohedral bnnn $(\mathrm{n}=12-28)$ cages. $J$. Chem. Phys. 128, 084306 (2008).

29. Nigam, S., Kulshreshtha, S. K. \& Majumder, C. Structural and magnetic isomers of $M(\mathrm{BN})_{36}$ and $M_{4}(\mathrm{BN})_{36}$ clusters $(M=\mathrm{Ti}, \mathrm{V}, \mathrm{Cr}, \mathrm{Mn}, \mathrm{Fe}, \mathrm{Co}$, $\mathrm{Ni}, \mathrm{Cu}$ ): An $a b$ initio density functional study. Phys. Rev. B 77, 075438 (2008).

30. Batista, R. J. C., Mazzoni, M. S. C. \& Chacham, H. Boron nitride fullerene $\mathrm{b}_{36} \mathrm{n}_{36}$ doped with transition metal atoms: first-principles calculations. Phys. Rev. B 75, 035417 (2007).

31. Wen, S.-H., Deng, W.-Q. \& Han, K.-L. Endohedral BN metallofullerene m@b36n36 complex as promising hydrogen storage materials. J. Phys. Chem. C 112, 12195-12200 (2008).

32. Ohta, Y. Possible mechanism of BN fullerene formation from a boron cluster: density-functional tight-binding molecular dynamics simulations. J. Comput. Chem. 37, 886-895 (2016).

33. Krstic, P. S., Han, L., Irle, S. \& Nakai, H. Simulations of the synthesis of boronnitride nanostructures in a hot, high pressure gas volume. Chem. Sci. 9, 3803-3819 (2018).

34. Ding, K.-W. et al. Experimental observation of $\operatorname{tin}(12)(+)$ cluster and theoretical investigation of its stable and metastable isomers. Chem. Sci. 6, 4723-4729 (2015).

35. Wang, Y. Maximum bonding fragment orbitals for deciphering complex chemical interactions. Phys. Chem. Chem. Phys. 20, 13792-13809 (2018).

36. Zhang, J.-X., Sheong, F. K. \& Lin, Z. Unravelling chemical interactions with principal interacting orbital analysis. Chem. Eur. J. 24, 9639-9650 (2018).

37. Wiberg, K. Application of the pople-santry-segal cndo method to the cyclopropylcarbinyl and cyclobutyl cation and to bicyclobutane. Tetrahedron 24, 1083-1096 (1968).

38. Slanina, Z. Multimolecular clusters: their isomerism and effective characteristics evaluated by quantum chemistry. Int. J. Quant. Chem. 16, 79-86 (1979).

39. Fathalizadeh, A., Pham, T., Mickelson, W. \& Zettl, A. Scaled synthesis of boron nitride nanotubes, nanoribbons, and nanococoons using direct feedstock injection into an extended-pressure, inductively-coupled thermal plasma. Nano Lett. 14, 4881-4886 (2014).

40. Huang, Y. \& Freiser, B. S. Externally bound metal ion complexes of buckminsterfullerene, mc60+, in the gas phase. J. Am. Chem. Soc. 113, 9418-9419 (1991).

41. Krachmalnicoff, A. et al. The dipolar endofullerene hf@c $\mathrm{c}_{60}$. Nat. Chem. 8, 953-957 (2016).
42. Zhang, R. et al. Synthesis of a distinct water dimer inside fullerene c70. Nat Chem. 8, 435-441 (2016)

43. Choi, H., Park, Y. C., Kim, Y.-H. \& Lee, Y. S. Ambient carbon dioxide capture by boron-rich boron nitride nanotube. J. Am. Chem. Soc. 133, 2084-2087 (2011).

44. Kumar, C. V. S. \& Subramanian, V. Can boron antisites of bnnts be an efficient metal-free catalyst for nitrogen fixation?-a DFT investigation. Phys. Chem. Chem. Phys. 19, 15377-15387 (2017).

45. Frisch, M. J. et al. Gaussian 16, Revision B.01 (Gaussian, Inc., Wallingford CT, 2016).

46. Grimme, S., Ehrlich, S. \& Goerigk, L. Effect of the damping function in dispersion corrected density functional theory. J. Comput. Chem. 32, 1456-1465 (2011)

47. Brinkmann, G. \& McKay, B.D. The program plantri, version 5.0. http://users cecs.anu.edu.au/bdm/plantri/ (2016).

48. Brinkmann, G., Friedrichs, O. D., Lisken, S., Peeters, A. \& Cleemput, N. V. $\mathrm{CaGe}-\mathrm{a}$ virtual environment for studying some special classes of plane graphs-an update. MATCH Commun. Math. Comput. Chem. 63, 533-552 (2010).

49. Wang, Y., Díaz-Tendero, S., Alcamí, M. \& Martín, F. Topology-based approach to predict relative stabilities of charged and functionalized fullerenes. J. Chem. Theory Comput. 14, 1791-1810 (2018).

50. Fowler, P. W., Horspool, D. \& Myrvold, W. Vertex spirals in fullerenes and their implications for nomenclature of fullerene derivatives. Chem. Eur. J. 13, 2208-2217 (2007).

\section{Acknowledgements}

Y.W. acknowledges the Thousand Talents Plan for Young Professionals of China.

\section{Author contributions}

Y.W. conceived the research and developed the computer codes. R.L. performed the DFT calculations and collected the data. Y.W. wrote the paper.

\section{Competing interests}

The authors declare no competing interests.

\section{Additional information}

Supplementary information is available for this paper at https://doi.org/10.1038/s41467 019-12877-0.

Correspondence and requests for materials should be addressed to Y.W.

Peer review information Nature Communications thanks Kyle Beran, Douglas Strout and the other anonymous reviewer(s) for their contribution to the peer review of this work. Peer reviewer reports are available.

Reprints and permission information is available at http://www.nature.com/reprints

Publisher's note Springer Nature remains neutral with regard to jurisdictional claims in published maps and institutional affiliations.

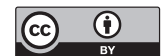

Open Access This article is licensed under a Creative Commons Attribution 4.0 International License, which permits use, sharing, adaptation, distribution and reproduction in any medium or format, as long as you give appropriate credit to the original author(s) and the source, provide a link to the Creative Commons license, and indicate if changes were made. The images or other third party material in this article are included in the article's Creative Commons license, unless indicated otherwise in a credit line to the material. If material is not included in the article's Creative Commons license and your intended use is not permitted by statutory regulation or exceeds the permitted use, you will need to obtain permission directly from the copyright holder. To view a copy of this license, visit http://creativecommons.org/ licenses/by/4.0/

(C) The Author(s) 2019 